\title{
Year-Long Trend in Glycated Hemoglobin Levels in Patients with Type 2 Diabetes during the COVID-19 Pandemic
}

\author{
Jonghwa Jin ${ }^{1}$, Seong Wook Lee ${ }^{1}$, Won-Ki Lee ${ }^{2}$, Jae-Han Jeon ${ }^{3}$, Jung-Guk Kim ${ }^{1}$, In-Kyu Lee ${ }^{1}$, Yeon-Kyung Choi ${ }^{3}$, \\ Keun-Gyu Park ${ }^{1}$ \\ ${ }^{1}$ Department of Internal Medicine, Kyungpook National University Hospital, School of Medicine, Kyungpook National \\ University; ${ }^{2}$ Center of Biostatistics, School of Medicine, Kyungpook National University; ${ }^{3}$ Department of Internal Medicine, \\ Kyungpook National University Chilgok Hospital, School of Medicine, Kyungpook National University, Daegu, Korea
}

It has been suggested that the coronavirus disease 2019 (COVID-19) pandemic has had a negative impact on glycemic control in patients with type 2 diabetes mellitus (T2DM). However, no study has examined yearly trends in glycated hemoglobin (HbA1c) levels after the start of the COVID-19 outbreak. Here, we performed a retrospective analysis of HbAlc concentrations during the early period of the COVID-19 outbreak (COVID-19 cohort) and then compared the yearly trend in the mean HbA1c level, along with fluctuations in HbAlc levels, with those during previous years (non-COVID-19 cohorts). We observed that the mean HbA1c level in patients with T2DM increased during the first 6 months of the COVID-19 outbreak. After 6 months, HbA1c levels in the COVID-19 cohort returned to levels seen in the non-COVID-19 cohorts. The data suggest that vulnerable patients with T2DM should be monitored closely during the early period of a pandemic to ensure they receive appropriate care.

Keywords: COVID-19; Physical distancing; Glycated hemoglobin A; Diabetes mellitus

\section{INTRODUCTION}

Coronavirus disease 2019 (COVID-19), caused by severe acute respiratory syndrome coronavirus 2 , has become a worldwide pandemic [1]. Beginning on February 18, 2020, the number of COVID-19 infected patients in Daegu increased rapidly during

Received: 14 June 2021, Revised: 15 August 2021, Accepted: 10 September 2021

Corresponding authors: Keun-Gyu Park

Department of Internal Medicine, Kyungpook National University Hospital,

School of Medicine, Kyungpook National University, 130 Dongdeok-ro, Jung-gu, Daegu 41944, Korea

Tel: +82-53-200-5505, Fax: +82-53-426-2046, E-mail: kpark@knu.ac.kr

Yeon-Kyung Choi

Department of Internal Medicine, Kyungpook National University Chilgok Hospital, School of Medicine, Kyungpook National University, 807 Hoguk-ro, Buk-gu, Daegu 41404, Korea

Tel: +82-53-200-5505, Fax: +82-53-426-2046, E-mail: ykchoi@knu.ac.kr the 1st month of the outbreak [2]. Strict social distancing began from March 23, 2020, and lasted until May 6, 2020 (Supplemental Fig. S1) [3].

This social distancing led to decreased physical activity and altered dietary habits in patients with diabetes [4]. These lifestyle changes caused a deterioration in metabolic status and gly-

\section{Copyright $\odot 2021$ Korean Endocrine Society}

This is an Open Access article distributed under the terms of the Creative Commons Attribution Non-Commercial License (https://creativecommons.org/ licenses/by-nc/4.0/) which permits unrestricted non-commercial use, distribution, and reproduction in any medium, provided the original work is properly cited. 
cemic control [5]. In a previous study, we showed that glycated hemoglobin (HbAlc) levels in patients with type 2 diabetes mellitus (T2DM) increased after implementation of social distancing during the early period of the COVID-19 pandemic [6]. However, long-term trends in HbAlc levels were not investigated.

In this study, we aimed to investigate the yearly trend in HbA1c levels among patients with T2DM during the pandemic and compare them with the trends during non-COVID-19 periods.

\section{METHODS}

This was a retrospective cohort study of consecutive patients with T2DM who visited the outpatient clinic at either Kyungpook National University Hospital (KNUH) or Kyungpook National University Chilgok Hospital (KNUCH) during November 2017 to February 2021. A flow chart of the study design is presented in Supplemental Fig. S2. The following data were collected retrospectively from medical records: age, sex, and $\mathrm{HbA1c}$ values. Patients aged $<19$ years, those who visited the clinics for the first time during Period 1 , and those who were diagnosed with COVID-19 or in close contact with COVID-19 patients were excluded. A total of 3,052 patients were enrolled. Patients were classified into three cohorts: a COVID-19 cohort
(2019 to 2021), non-COVID-19 cohort 1 (2018 to 2020), and non-COVID cohort 2 (2017 to 2019). Changes in HbAlc $(\triangle \mathrm{HbAlc})$ were calculated by subtracting the value measured during each period from the value at baseline (Period 1); the $\triangle \mathrm{HbAlc}$ value from baseline was compared with those in the non-COVID-19 cohorts (control groups).

Differences in the mean $\mathrm{HbA} 1 \mathrm{c}$ for each period, and of $\triangle \mathrm{HbA} 1 \mathrm{c}$ among the three cohorts, were examined using analysis of variance. An $\triangle \mathrm{HbA} 1 \mathrm{c}$ values were estimated after adjusting for multiple confounding factors using analysis of covariance. $P<0.05$ was considered significant. Statistical analysis was performed using R studio 1.4.1106 for Windows (R Studio Inc., Boston, MA, USA). The study was approved by the Institutional Review Boards of KNUH (2020-05-063-001). The necessity for informed consent was waived by the ethics boards of the hospitals because of the retrospective study design.

\section{RESULTS}

There were no significant differences among the three cohorts with respect to age, sex, and HbA1c at baseline (Supplemental Table S1). The mean HbA1c in the COVID-19 cohort during Period 1 did not differ statistically from that in the other cohorts (7.23\% $\pm 1.18 \%$ for the COVID-19 cohort, $7.19 \% \pm 0.97 \%$ for non-COVID-19 cohort 1 , and $7.25 \% \pm 1.14 \%$ for non-COV-

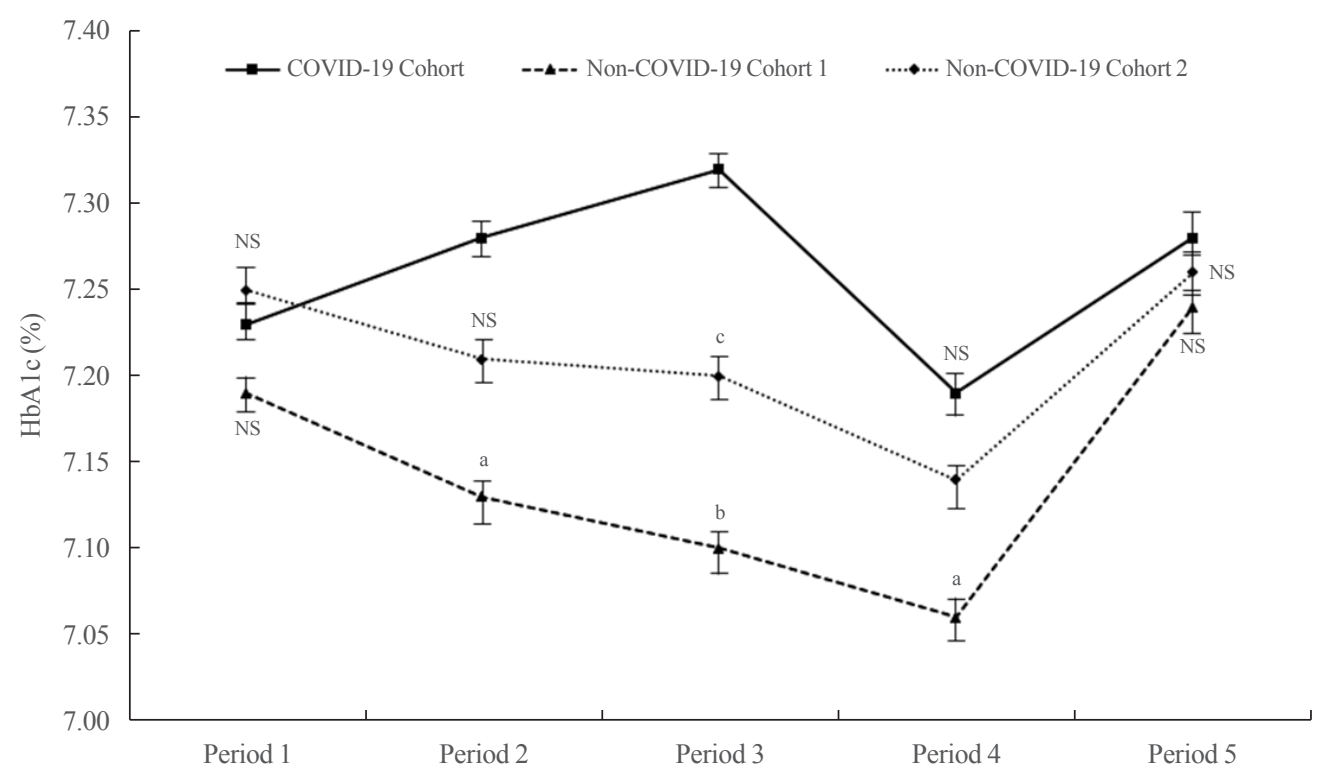

Fig. 1. Changes in glycated hemoglobin (HbAlc) levels from Period 1 to Period 5 in all three cohorts. Values are expressed as the mean \pm standard error. There were no significant differences between non-coronavirus disease 2019 (COVID-19) cohort 1 and cohort 2 during any period. NS, not significant. ${ }^{a} P<0.01$, COVID-19 cohort vs. non-COVID-19 cohort 1 ; ${ }^{\mathrm{b}} P<0.05$, COVID-19 cohort vs. non-COVID-19 cohort $1 ;{ }^{\mathrm{c}} P<0.01$, COVID-19 cohort vs. non-COVID-19 cohort 2. 
ID-19 cohort 2; $P=0.579$ ) (Fig. 1). In Period 2, the mean HbA1c in the COVID-19 cohort was higher than that in the non-COVID-19 cohort $1(7.28 \% \pm 1.45 \%$ vs. $7.13 \% \pm 0.93 \%$, $P<0.05)$ and cohort $2(7.28 \% \pm 1.45 \%$ vs. $7.21 \% \pm 1.07 \%$, $P=0.423)$. In Period 3, the mean HbA1c in the COVID-19 cohort was significantly higher than that in the non-COVID-19 cohort $1(7.32 \% \pm 1.36 \%$ vs. $7.11 \% \pm 0.80 \%, P<0.01)$ and cohort $2(7.32 \% \pm 1.36 \%$ vs. $7.17 \% \pm 1.19 \%, P<0.05)$. The mean $\mathrm{HbA1c}$ levels among the three cohorts tended to decrease during Period 4. Nonetheless, the mean HbAlc level in the COVID-19 cohort remained higher than that in the non-COVID-19 cohort $1(7.19 \% \pm 1.28 \%$ vs. $7.06 \% \pm 1.00 \%, P<0.05)$ and cohort 2 (7.19\% $\pm 1.28 \%$ vs. $7.13 \% \pm 1.10 \%, P=0.492)$. In Period 5 , levels tended to increase in all three cohorts, but there were no significant differences $(7.28 \% \pm 1.45 \%$ in the COVID-19 cohort, $7.24 \% \pm 0.97 \%$ in non-COVID-19 cohort 1 , and $7.27 \% \pm$ $1.28 \%$ in non-COVID-19 cohort $2 ; P=0.720$ ).

After adjusting for sex and baseline HbAlc levels, the $\triangle \mathrm{HbA} 1 \mathrm{c}$ from baseline to Period 2 was higher in the COVID-19 cohort than that in the non-COVID-19 cohort 1 and cohort 2 ( $P<0.01$ and $P<0.05$, respectively) (Fig. 2). The adjusted $\triangle \mathrm{HbA} 1 \mathrm{c}$ level from baseline to Period 3 in the COVID-19 cohort was greater than that in the non-COVID-19 cohort 1 and cohort 2 ( $P<0.01$ and $P<0.01$, respectively). The $\Delta \mathrm{HbA} 1 \mathrm{c}$ from baseline to Period 4 fell in all three groups. Nonetheless, the degree of $\mathrm{HbAlc}$ reduction compared with baseline in the COV-
ID-19 cohort was lower than that in the non-COVID-19 cohort $1(P<0.05)$. There was no significant difference between the groups with respect to the adjusted $\triangle \mathrm{HbAlc}$ from baseline to Period $5(P=0.828)$.

\section{DISCUSSION}

The data show that the mean HbAlc level in patients with T2DM increased during the first 6 months of the COVID-19 outbreak. After 6 months, the trend in HbA1c levels showed a pattern similar to that observed in the non-COVID-19 cohorts.

Previous studies demonstrate that prolonged periods of physical inactivity and modified dietary patterns caused by social distancing contribute to poorer glycemic outcomes [5,7,8]. A recent study showed that patients with diabetes are more vulnerable than people without diabetes to psychological distress related to social distancing [9]. Moreover, the negative association between recent severe stress and glycemic control among patients with diabetes support the finding that lifestyle changes and/or mental stress during social distancing had a harmful impact on glycemic control during the first 6 months of the outbreak.

A seasonal variation in glucose homeostasis in patients with diabetes has been reported in various geographic regions [1,1013]. HbA1c levels are reported to decrease in the summer/fall and increase in the winter [11-13], similar to the results of the

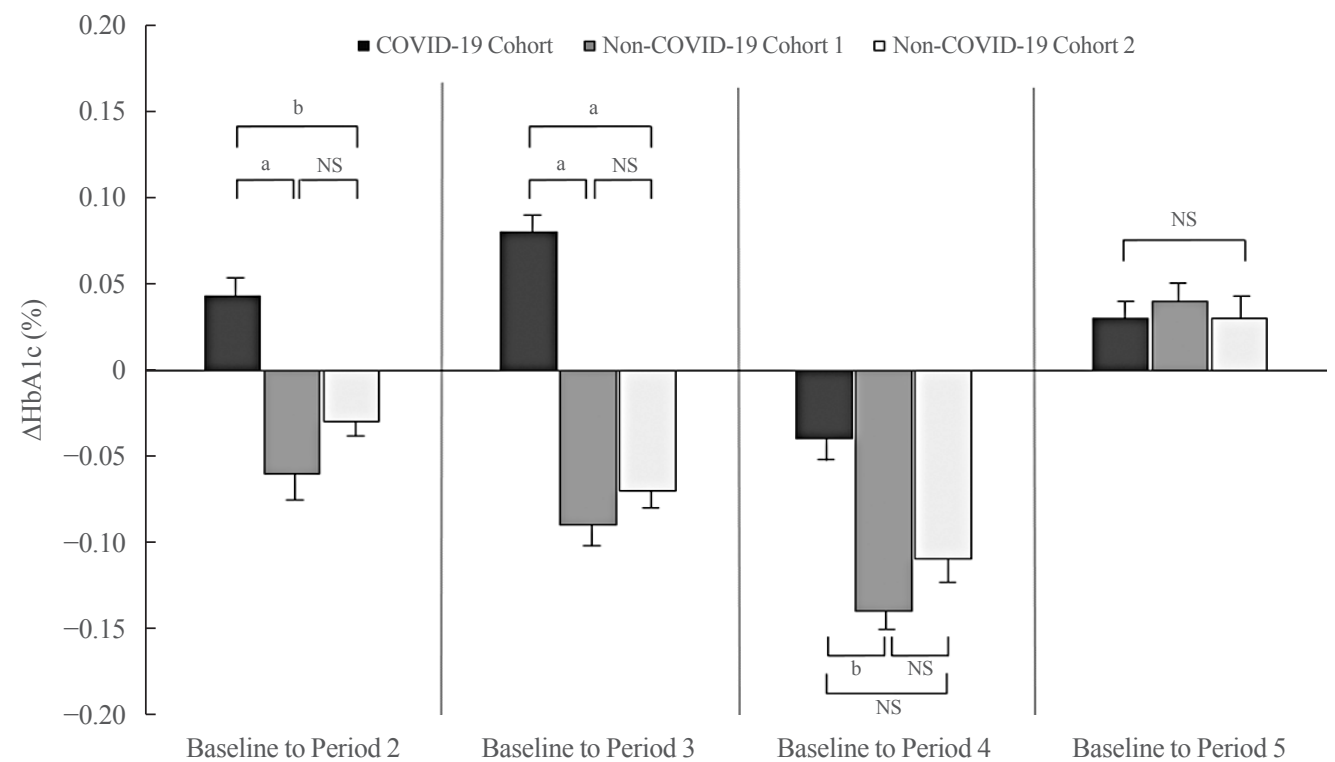

Fig. 2. Changes in glycated hemoglobin (HbA1c) levels (data adjusted for baseline $\mathrm{HbA}$ 1c level and sex). $\Delta \mathrm{HbA} \mathrm{c}$ from baseline period to each period is shown for all three cohorts. Values are expressed as the mean \pm standard error. COVID-19, coronavirus disease 2019; NS, not significant. ${ }^{\mathrm{a}} P<0.01 ;{ }^{\mathrm{b}} \mathrm{P}<0.05$. 
non-COVID-19 cohort in our study. However, we found that the seasonal variation in HbA1c levels did not occur in the COVID-19 cohort during the early stages of the COVID-19 pandemic. The trend in the COVID-19 cohort was analogous to those in the other cohorts for the last 6 months of the year. Even in the COVID-19 cohort, the mean HbA1c level returned to that observed before the beginning of the crisis.

A recent study reported that age, duration of education, frequency of self-monitoring of blood glucose, and medication compliance are related to glycemic control, whereas structure of diet, body mass index, duration of diabetes, exercise, sleep, hypoglycemia, and anxiety are not related with glycemic control during the COVID-19 pandemic [14]. Since the participants were recruited retrospectively, we could not analyze the association between these contributing factors and $\mathrm{HbA} 1 \mathrm{c}$ levels or compare them between COVID-19 and non-COVID-19 cohorts. To clinically strengthen our findings, further in-depth analysis considering various clinical variables will be required. Another limitation is that our findings may not be generalizable to other areas because Daegu has been relatively successful at controlling infections, with a rate below 100 cases per day since March 2020. Additionally, this retrospective study was conducted in only two centers, and the retrospective nature may introduce some bias with regard to patient selection.

Despite these limitations, we believe that the findings of the study are meaningful. To the best of our knowledge, this report is the first to show that social distancing has an impact on glycemic control in patients with T2DM, and that this impact persisted for more than 6 months. This suggests that careful attention must be paid to vulnerable patients with T2DM during the early periods of a pandemic such as that caused by COVID-19.

\section{CONFLICTS OF INTEREST}

No potential conflict of interest relevant to this article was reported.

\section{ACKNOWLEDGMENTS}

This work was supported by a Biomedical Research Institute grant from Kyungpook National University Hospital (2015).

\section{AUTHOR CONTRIBUTIONS}

Conception or design: J.J., K.G.P. Acquisition, analysis, or interpretation of data: J.J., S.W.L., W.K.L., Y.K.C., K.G.P. Draft- ing the work or revising: J.J., J.H.J., Y.K.C., K.G.P. Final approval of the manuscript: J.J., S.W.L, W.K.L., J.H.J., J.G.K., I.K.L., Y.K.C., K.G.P.

\section{ORCID}

Jonghwa Jin https://orcid.org/0000-0002-9286-3190

Yeon-Kyung Choi https://orcid.org/0000-0003-0996-6437

Keun-Gyu Park https://orcid.org/0000-0002-8403-1298

\section{REFERENCES}

1. Sharma A, Tiwari S, Deb MK, Marty JL. Severe acute respiratory syndrome coronavirus-2 (SARS-CoV-2): a global pandemic and treatment strategies. Int J Antimicrob Agents 2020;56:106054.

2. Bae TW, Kwon KK, Kim KH. Mass infection analysis of COVID-19 using the SEIRD model in Daegu-Gyeongbuk of Korea from April to May, 2020. J Korean Med Sci 2020; 35:e317.

3. Park IN, Yum HK. Stepwise strategy of social distancing in Korea. J Korean Med Sci 2020;35:e264.

4. Ruiz-Roso MB, Knott-Torcal C, Matilla-Escalante DC, Garcimartin A, Sampedro-Nunez MA, Davalos A, et al. COVID-19 lockdown and changes of the dietary pattern and physical activity habits in a cohort of patients with type 2 diabetes mellitus. Nutrients 2020;12:2327.

5. Tanaka N, Hamamoto Y, Kurotobi Y, Yamasaki Y, Nakatani S, Matsubara M, et al. Lifestyle changes as a result of COVID-19 containment measures: bodyweight and glycemic control in patients with diabetes in the Japanese declaration of a state of emergency. J Diabetes Investig 2021;12:1718-22.

6. Park SD, Kim SW, Moon JS, Lee YY, Cho NH, Lee JH, et al. Impact of social distancing due to coronavirus disease 2019 on the changes in glycosylated hemoglobin level in people with type 2 diabetes mellitus. Diabetes Metab J 2021;45:109-14.

7. Di Renzo L, Gualtieri P, Pivari F, Soldati L, Attina A, Cinelli $\mathrm{G}$, et al. Eating habits and lifestyle changes during COVID-19 lockdown: an Italian survey. J Transl Med 2020;18: 229.

8. Ghosh A, Arora B, Gupta R, Anoop S, Misra A. Effects of nationwide lockdown during COVID-19 epidemic on lifestyle and other medical issues of patients with type 2 diabetes in north India. Diabetes Metab Syndr 2020;14:917-20.

9. Alessi J, de Oliveira GB, Franco DW, Brino do Amaral B, 
Becker AS, Knijnik CP, et al. Mental health in the era of COVID-19: prevalence of psychiatric disorders in a cohort of patients with type 1 and type 2 diabetes during the social distancing. Diabetol Metab Syndr 2020;12:76.

10. Dasgupta K, Joseph L, Pilote L, Strachan I, Sigal RJ, Chan C. Daily steps are low year-round and dip lower in fall/winter: findings from a longitudinal diabetes cohort. Cardiovasc Diabetol 2010;9:81.

11. Gikas A, Sotiropoulos A, Pastromas V, Papazafiropoulou A, Apostolou O, Pappas S. Seasonal variation in fasting glucose and $\mathrm{HbAlc}$ in patients with type 2 diabetes. Prim Care Diabetes 2009;3:111-4.
12. Kim YJ, Park S, Yi W, Yu KS, Kim TH, Oh TJ, et al. Seasonal variation in hemoglobin alc in Korean patients with type 2 diabetes mellitus. J Korean Med Sci 2014;29:550-5.

13. Pereira MT, Lira D, Bacelar C, Oliveira JC, de Carvalho AC. Seasonal variation of haemoglobin A1c in a Portuguese adult population. Arch Endocrinol Metab 2015;59:231-5.

14. Tao J, Gao L, Liu Q, Dong K, Huang J, Peng X, et al. Factors contributing to glycemic control in diabetes mellitus patients complying with home quarantine during the coronavirus disease 2019 (COVID-19) epidemic. Diabetes Res Clin Pract 2020;170:108514. 